\title{
LOWER AND UPPER SOLUTIONS FOR SINGULAR DERIVATIVE DEPENDENT DIRICHLET PROBLEM
}

\section{DE COSTER}

Abstract. In this work, we consider the Dirichlet problem

$$
\begin{aligned}
& u^{\prime \prime}+f\left(t, u, u^{\prime}\right)=0, \\
& u(a)=0, \quad u(b)=0,
\end{aligned}
$$

with $f$ singular at $t=a, t=b$ and for $u=0$ and extend previous results concerning the case $f$ independent of $u^{\prime}$. To this aim we extend the lower and upper solution method in order to work with solutions in $W^{1,1}(a, b) \cap W_{l o c}^{2,1}(a, b)$ as well as with lower and upper solutions having unbounded derivatives.

Mathematics subject classification (2000): 34B16, 34B18, 34C11.

Key words and phrases: singular boundary value problem, lower and upper solutions, derivative dependent nonlinearity.

\section{REFERENCES}

[1] K. AKO, Subfunctions for ordinary differential equations II, Funkcialaj Ekvacioj 10(1967), $145-162$.

[2] K. AKO, Subfunctions for ordinary differential equations V, Funkcialaj Ekvacioj 12(1969), 239-249.

[3] J. V. BAXLEY, A singular nonlinear boundary value problem: membrane response of a spherical cap, SIAM J. Appl. Math. 48(1988), 497-505.

[4] S. BeRnsteIn, Sur certaines équations différentielles ordinaires du second ordre, C.R.A.S. Paris 138(1904), 950-951.

[5] L. E. Bobisud, D. O'REGAN AND W. D. RoyAlty, Solvability of some nonlinear boundary value problem, Nonlinear Anal. T.M.A. 12(1988), 855-869.

[6] L. Boccardo, F. Murat AND J. P. PuEL, Résultats d'existence pour certains problèmes elliptiques quasilinéaires, Ann. Scuola Norm. Sup. Pisa 11(1984), 213-225.

[7] A. CAllegari AND A. NACHMAN, Some singular nonlinear differential equations arising in boundary layer theory, J. Math. Anal. Appl. 64(1978), 96-105.

[8] B. Dacorogna, Direct methods in the calculus of variations, Springer-Verlag, Berlin 1989.

[9] H. EPHESER, Über die existenz der lösungen von randwertaufgaben mit gewöhnlichen, nichtlinearen differentialgleichungen zweiter ordnung, Math. Zeitschr. 61(1955), 435-454.

[10] C. FABRY AND P. HABETS, Upper and lower solutions for second-order boundary value problems with nonlinear boundary conditions, Nonlinear Anal. T.M.A. 10(1986), 985-1007.

[11] J. A. Gatica, V. Oliker AND P. Waltman, Singular nonlinear boundary value problems for second order ordinary differential equations, J. Diff. Equ. 79(1989), 62-78.

[12] V. V. GUDKOV AND A. J. LEPIN, On necessary and sufficient conditions for the solvability of certain boundary-value problems for a second-order ordinary differential equation, Dokl. Akad. Nauk SSSR 210(1973), 800-803.

[13] P. HABETS AND F. ZANOLIN, Upper and lower solutions for a generalized Emden-Fowler equation, J. Math. Anal. Appl. 181 (1994), 684-700.

[14] P. HABETS AND F. ZANOLIN, Positive solutions for a class of singular boundary value problem, Boll. U.M.I. (7) 9-A(1995), 273-286. 
[15] J. JANUS AND J. MYJAK, A generalized Emden-Fowler equation with a negative exponent, Nonlinear Anal. T.M.A. 23 (1994), 953-970.

[16] I. T. KIGURADZE, A priori estimates for derivatives of bounded functions satisfying second-order differential inequalities, Differentsial'nye Uravneniya 3(1967), 1043-1052.

[17] I. T. KiguRADZE, Some singular boundary value problems for ordinary nonlinear second order differential equations, Differentsial'nye Uravneniya 4(1968), 1753-1773.

[18] I. T. KiguRADZE, On a singular boundary value problem, J. Math. Anal. Appl. 30(1970), 475-489.

[19] I. T. KIGURADZE AND B. L. SHEKHTER, Singular boundary-value problems for ordinary second-order differential equations, Itogi Nauki i Tekhniki, Seriya Sovremennye Problemy Matematiki, Noveishie Dostizheniya 30 (1987), 105-201, translated in J. Soviet Math. 43(1988), 2340-2417.

[20] A. G. LOMTATIDZE, Positive solutions of boundary value problems for second order ordinary differential equations with singular points, Differentsial'nye Uravneniya 23(1987), 1685-1692.

[21] R. D. MOYER, Second order differential equations of monotonic type, J. Diff. Equ. 2(1966), 281-292.

[22] A. NACHMAN AND A. CALLEGARI, A nonlinear boundary value problem in the theory of pseudoplastic fluids, SIAM J. Appl. Math. 38(1980), 275-281.

[23] M. Nagumo, Über die differentialgleichung $y^{\prime \prime}=f\left(t, y, y^{\prime}\right)$, Proc. Phys-Math. Soc. Japan 19(1937), 861-866.

[24] M. NAGUMo, $y^{\prime \prime}=f\left(t, y, y^{\prime}\right)$ no kyôkaichi mondai ni tsuite I, II, Kansû Hôteishiki 17(1942) 36-46, $50-52$.

[25] M. Nagumo, On principally linear elliptic differential equations of the second order, Osaka Math. J. 6(1954), 207-229.

[26] E. PICARD, Sur l'application des méthodes d'approximations successives à l'étude de certaines équations différentielles ordinaires, J. de Math. 9(1893), 217-271.

[27] G. PRODI, Teoremi di esistenza per equazioni alle derivate parziali non lineari di tipo parabolico, Nota I e II, Rend. Ist. Lombardo 86(1953), 1-47.

[28] A. RosenBlatT, Sur les théorèmes de M. Picard dans la théorie des problèmes aux limites des équations différentielles ordinaires non linéaires, Bull. Sc. Math. 57(1933), 100-106.

[29] F. Z. SADYRBAEV, Lyapunov functions and the solvability of the first boundary-value problem for ordinary second-order differential equations, Diff. Equ. 16(1980), 387-391.

[30] G. SCORZA DRAGONI, Il problema dei valori ai limiti studiato in grande per gli integrali di una equazione differenziale del secondo ordine, Giornale di Mat. (Battaglini) 69(1931), 77-112.

[31] G. SCORZA DRAGONI, Intorno a un criterio di esistenza per un problema di valori ai limiti, Rend. Semin. R. Accad. Naz. Lincei 28(1938), 317-325.

[32] S. D. TALIAFERRO, A nonlinear singular boundary value problem, Nonlinear Anal. T.M.A. 3(1979), 897-904.

[33] A. TINEO, Existence theorems for a singular two-point Dirichlet problem, Nonlinear Anal. T.M.A. 19(1992), 323-333.

[34] M. X. WANG, J. J. NiETO AND A. CABADA, Monotone method for nonlinear second order periodic boundary value problem with Carathéodory functions, Ann. Pol. Math. 58(1993), 221-235.

[35] Zongming GuO, Solvability of some singular nonlinear boundary value problems and existence of positive radial solutions of some singular elliptic problems, Nonlinear Anal. T.M.A. 16(1991), 781-790. 